\title{
Automated High-Throughput Permethylation for Glycosylation Analysis of Biologics using MALDI-TOF-MS
}

Archana Shubhakar ${ }^{\dagger},{ }^{*}{ }^{*}$ Radoslaw P. Kozak, ${ }^{\dagger}$ Karli R. Reiding, ${ }^{\ddagger}$ Louise Royle, ${ }^{\dagger}$ Daniel I.R. Spencer, ${ }^{\dagger}$ Daryl L. Fernandes, ${ }^{\dagger}$ Manfred Wuhrer ${ }^{\ddagger}, \S$

† Ludger Ltd, Culham Science Centre, Abingdon, Oxfordshire, UK;

¥ Leiden University Medical Center, Center for Proteomics and Metabolomics, Leiden, The Netherlands;

$\S$ Division of BioAnalytical Chemistry, VU University Amsterdam, De Boelelaan 1083, 1081 HV Amsterdam, The Netherlands

Corresponding Author-*Email: archana.shubhakar@ludger.com Tel: +44 1865 408554; Fax: +44 8701634620

\section{Supporting Information}




\section{Contents of Supporting Information}

Slides 3, 4, 5 - Detailed experimental protocols section.

Figure S-1: Linear regression plot of eight major N-glycans from human IgG.

Figure S-2: Stacked MALDI-TOF-MS of permethylated human IgG illustrating working range.

Figure S-3: MALDI-TOF-MS of permethylated human IgG compared with water blank depicting specificity.

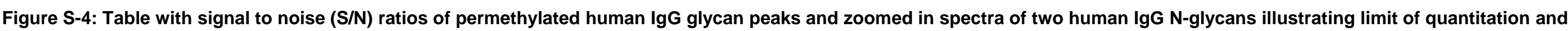
detection.

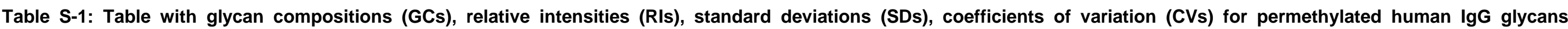
compared with 2-AB labeled glycans.

Table S-2: Table with GCs, RIs, SDs, CVs for permethylated human IgG sample for intraday variability.

Figure S-5: Table and linear regression plot for depicting interday variability.

Figure S-6: MALDI-TOF-MS spectra of In-solution and HT permethylation of N-glycans from human IgG.

Figure S-7: Comparison of relative intensities of N-linked glycans from A2G2S2 and A2G2S1 glycan standards using MALDI-TOF-MS and HILIC UHPLC.

Figure S-8A: MALDI-TOF-MS of permethylated N-glycans from IgG1 mAb.

Figure S-8B: UHPLC profile of procainamide labeled N-glycans from IgG1 mAb.

Table S-3: Table with bioreactor conditions, RIs, SDs, CVs for permethylated and 2-AB labeled IgG4 mAb.

Figure S-9: Typical permethylated MALDI-TOF-MS and chromatogram of 2-AB labeled IgG4 mAb trace.

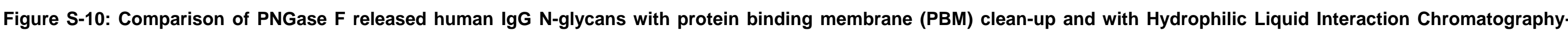
Solid Phase extraction (HILIC SPE) clean-up.

Figure S-11: Comparison of PNGase F released human IgG N-glycans with and without HILIC SPE clean-up.

Figure S-12: Line chart showing the efficiency of permethylation over two cycles of derivatization.

Figure S-13: Histogram comparing the RIs of major 7 N-linked glycans from rhEPO. 


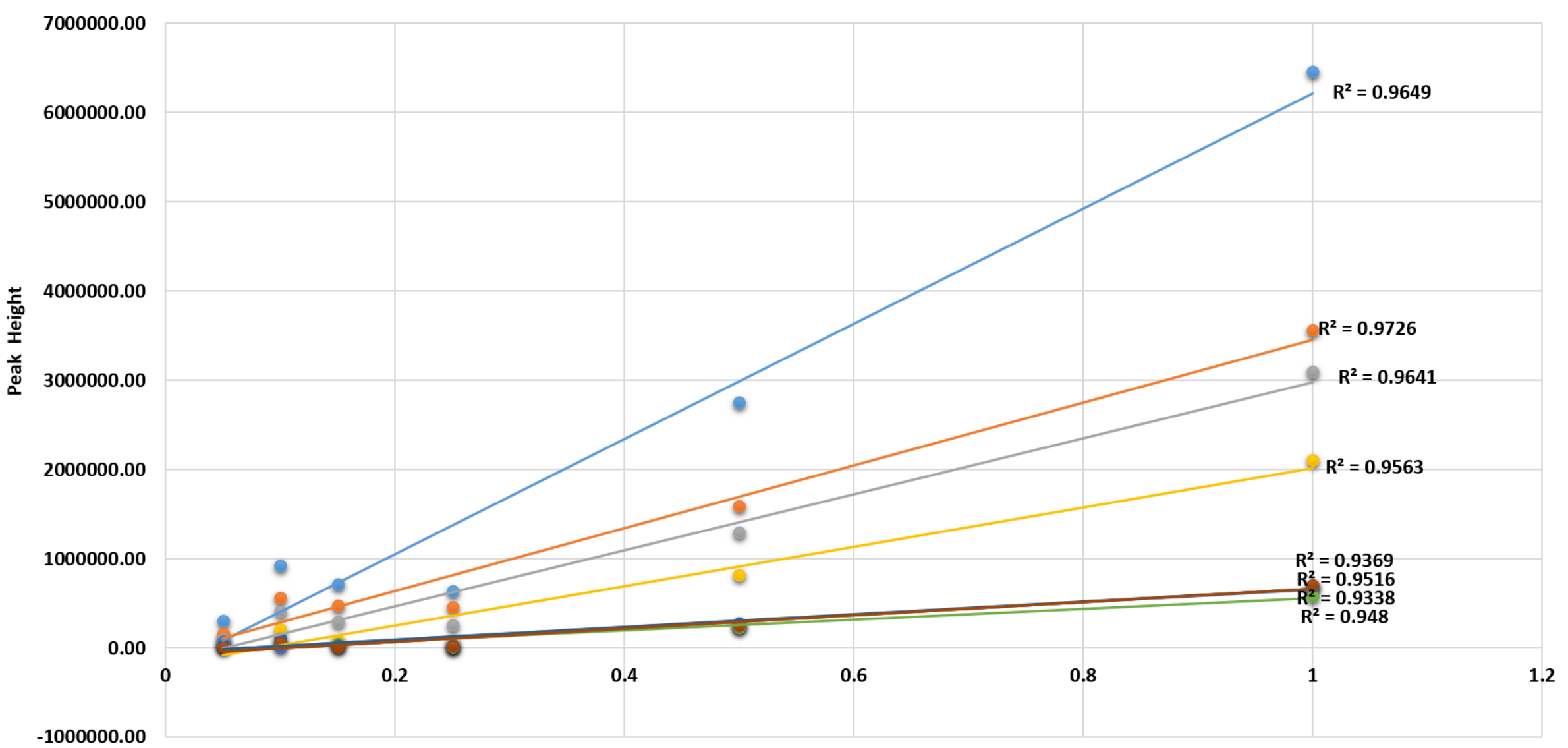

IgG $\mathrm{N}$-glycan peak concentration in $\mu \mathrm{g}$

Figure S-1: Triplicate samples of $\mathrm{lgG}$ glycoprotein from different starting material concentrations $(100 \mu \mathrm{g}, 50 \mu \mathrm{g}, 25 \mu \mathrm{g}, 15 \mu \mathrm{g}, 10 \mu \mathrm{g}$ and $5 \mu \mathrm{g})$ were released, enriched and permethylated using the robot. Concentrations of $(1 \mu \mathrm{g}, 0.5 \mu \mathrm{g}, 0.25 \mu \mathrm{g}, 0.15 \mu \mathrm{g}, 0.1 \mu \mathrm{g}$ and $0.05 \mu \mathrm{g})$ were spotted on the MALDI target and the peak intensities were plotted for eight major $\mathrm{N}$-glycan peaks. A visual evaluation of the analyte signals as a function of the concentrations for $\operatorname{lgG~N-glycans,~is~shown~in~this~linear~regression~plot~to~access~linearity~as~a~validation~parameter.~}$ 

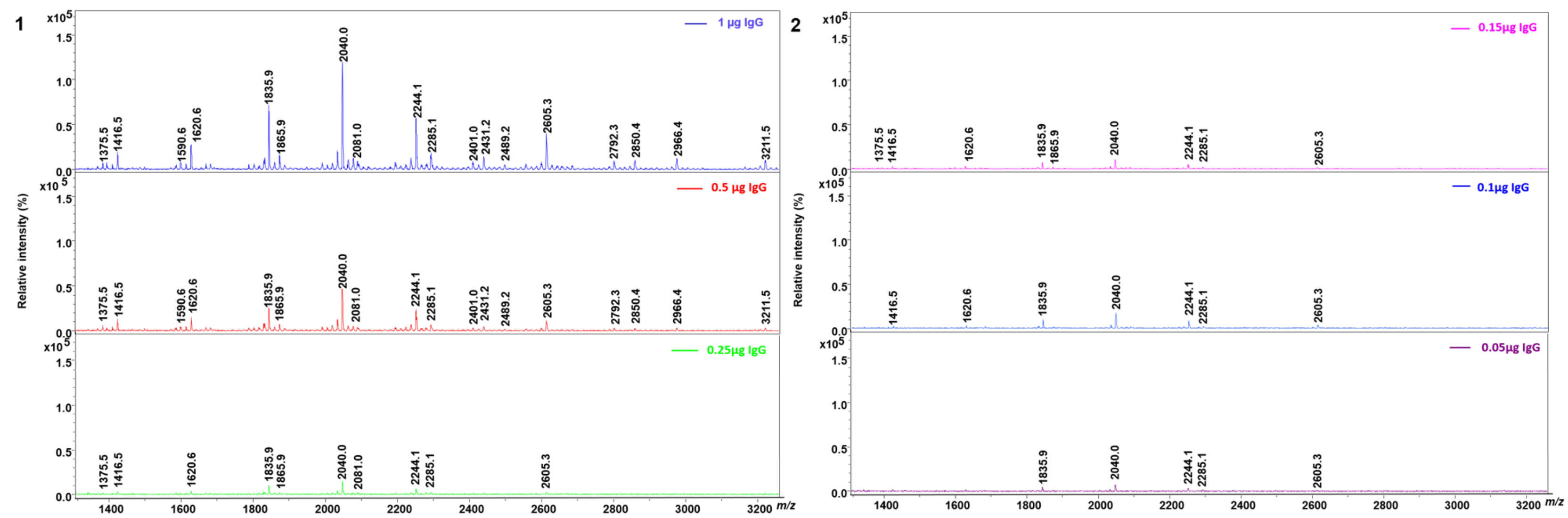

Figure S-2: Figure represents the comparison of relative intensities of permethylated N-linked glycans from released and enriched lgG glycoprotein for different concentrations spotted on the MALDI target $(1 \mu \mathrm{g}, 0.5 \mu \mathrm{g}, 0.25 \mu \mathrm{g}, 0.15 \mu \mathrm{g}, 0.1 \mu \mathrm{g}$ and $0.05 \mu \mathrm{g})$. As the concentration of lgG decreases, the intensity of the signal of glycans in $\mathrm{m} / z$ range of 1400 to $3200 \mathrm{decreases}$, where $Y$ axis is normalized.(1) Stacked MALDI-TOF-MS of permethylated human IgG showing sample concentration of $1 \mu \mathrm{g}, 0.5 \mu \mathrm{g}$ and $0.25 \mu \mathrm{g}$. (2) Stacked MALDI-TOF-MS of permethylated human IgG showing sample concentration of $0.15 \mu \mathrm{g}, 0.1 \mu \mathrm{g}$ and $0.05 \mu \mathrm{g}$. (The mass values shown in all the spectra are [M+Na]+ of permethylated glycans). 


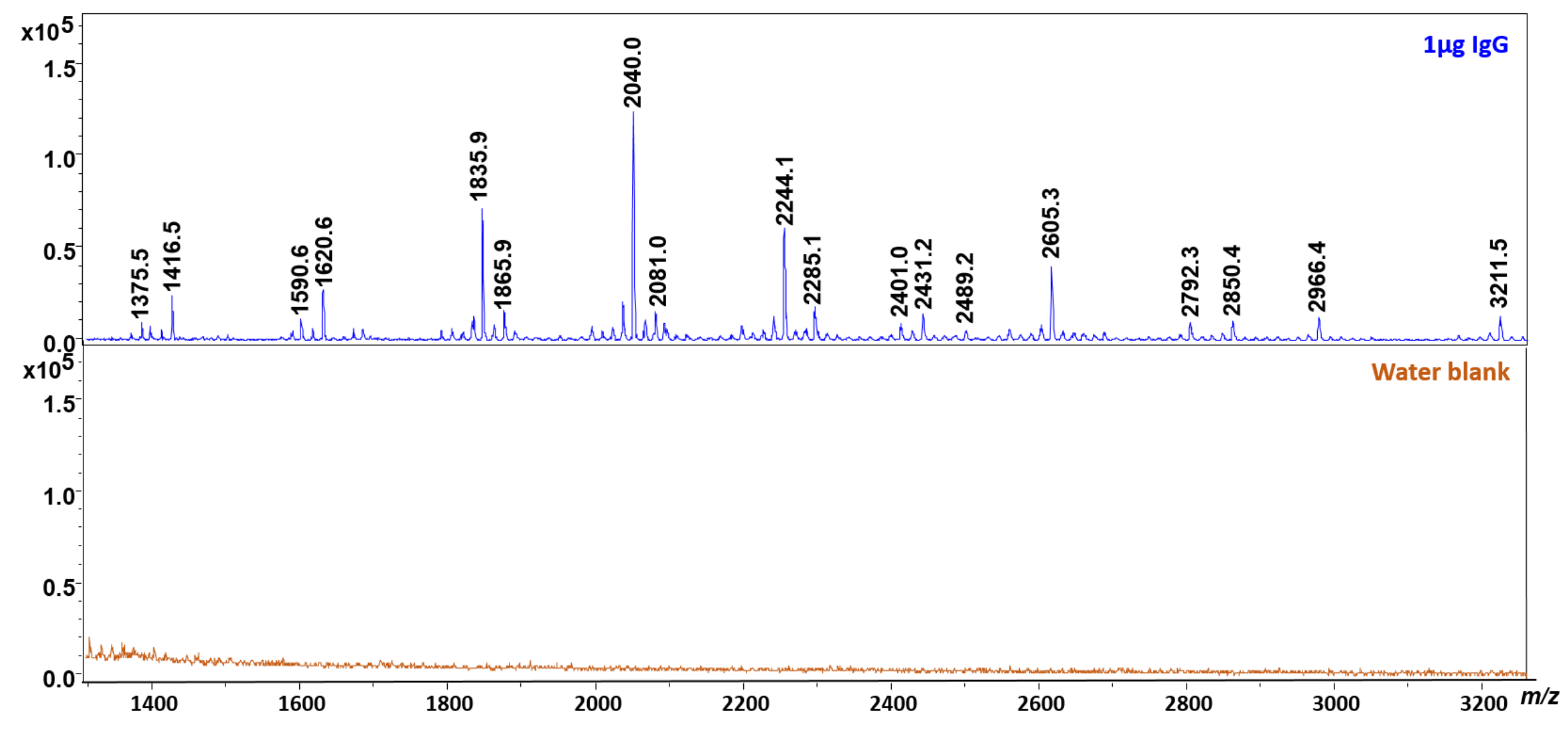

Figure S-3: MALDI-TOF-MS of permethylated N-glycans from human IgG compared with water blank (negative control) analyzed in parallel where the water blank underwent the same sample processing as the human IgG sample. $Y$ axis is normalized to show that the negative control components do not interfere with permethylated glycans and demonstrate specificity of the method. 
A

\begin{tabular}{|c|c|c|c|c|c|c|c|}
\hline \multicolumn{7}{|c|}{ S/N ratios of permethylated MALDI-TOF-MS of human IgG N-glycans } \\
\hline $\begin{array}{c}\text { Glycan } \\
\text { composition }\end{array}$ & $\begin{array}{c}\mathrm{m} / \mathrm{z} \\
{[\mathrm{M}+\mathrm{Na}]^{+}}\end{array}$ & $1 \mu \mathrm{gg}$ & $0.5 \mu \mathrm{g}$ & $0.25 \mu \mathrm{g}$ & $0.15 \mu \mathrm{g}$ & $0.1 \mu \mathrm{g}$ & $0.05 \mu \mathrm{g}$ \\
\hline H3N4F1 & 1835.9 & 104 & 58 & 17 & 19 & 19 & 8 \\
\hline H4N4F1 & 2040.0 & 179 & 92 & 24 & 27 & 32 & 10 \\
\hline H5N4F1 & 2244.1 & 84 & 42 & 10 & 12 & 14 & 5 \\
\hline H4N5F1 & 2285.1 & 25 & 12 & 4 & 4 & 4 & 3 \\
\hline H5N4F1S1 & 2605.3 & 58 & 24 & 4 & 4 & 8 & 2 \\
\hline
\end{tabular}

B

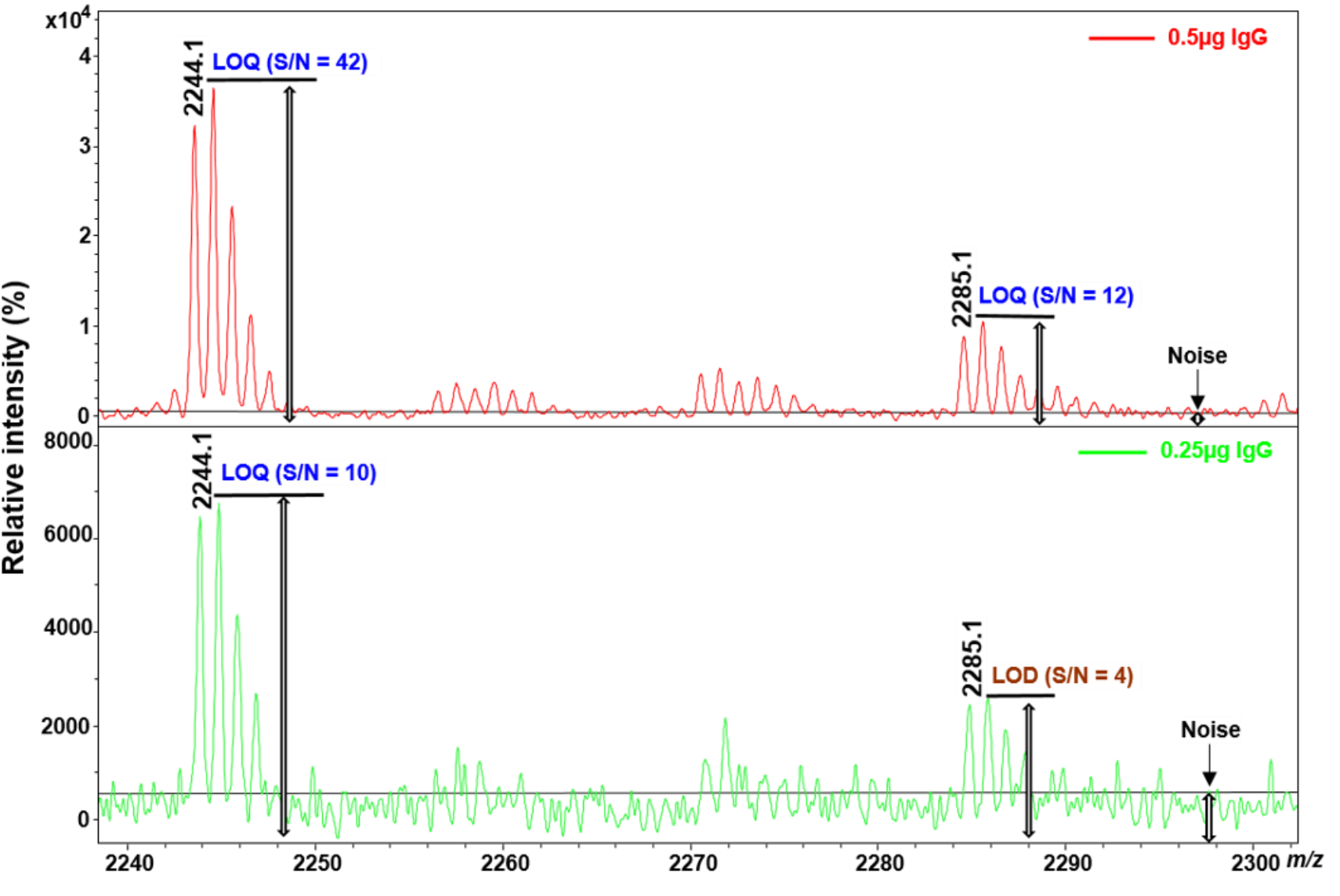

Figure S-4: (A) Table with signal to noise $(\mathrm{S} / \mathrm{N})$ ratios of permethylated human lgG glycan peaks above $5 \%$ relative areas. Samples with different concentrations $(1 \mu \mathrm{g}, 0.5 \mu \mathrm{g}, 0.25 \mu \mathrm{g}$, $0.15 \mu \mathrm{g}, 0.1 \mu \mathrm{g}$ and $0.01 \mu \mathrm{g}$ ) were spotted on the MALDI target, the $\mathrm{S} / \mathrm{N}$ ratios gradually decrease with decrease in concentration which is evident from the table. S/N ratios in blue are acceptable for LOQ, brown are acceptable for LOD and not quantifiable and red S/N ratio are not acceptable for quantitation or for detection. (B) Zoomed in spectra of two human IgG Nglycans ( $\mathrm{m} / \mathrm{z}$ values of 2244.1 and 2285.1 ) at $0.5 \mu \mathrm{g}$ and $0.25 \mu \mathrm{g}$ concentration illustrating LOQ and LOD determination using S/N ratios. Both peaks with $\mathrm{m} / z$ values of 2244.1 and 2285.1 shown in the red spectrum $(0.5 \mu \mathrm{g}$ concentration) are quantifiable as they have $\mathrm{S} / \mathrm{N}$ ratios of 42 and 12 respectively. While, the peak 2244.1 is quantifiable ( $\mathrm{S} / \mathrm{N}=10)$ and peak 2285.1 from the green spectrum ( $0.25 \mu \mathrm{g}$ concentration) is only acceptable for detection and it is not quantifiable as the $\mathrm{S} / \mathrm{N}$ is 4 .

Note: $L O Q=10: 1$ and $L O D=3: 1$ of $S / N$ ratio. 
Table S-1: Peak numbers, glycan composition, relative intensities (RIs), standard deviation (SD) and coefficients of variation (CVs) for 2-AB labeled (HILIC UHPLC) data and permethylated (MALDI-TOF-MS) data for N-glycans from human IgG.

\begin{tabular}{|c|c|c|c|c|c|c|c|c|c|}
\hline \multirow{2}{*}{ Peak no. } & \multirow{2}{*}{$\begin{array}{c}\text { Glycan } \\
\text { composition }\end{array}$} & \multicolumn{4}{|c|}{$\begin{array}{l}\text { 2-AB labeled } \\
\text { HILIC- UHPLC }\end{array}$} & \multicolumn{4}{|c|}{$\begin{array}{l}\text { Permethylated } \\
\text { MALDI-TOF-MS } \\
\end{array}$} \\
\hline & & $\begin{array}{l}\text { Glucose Units } \\
\text { (GU) }\end{array}$ & $\begin{array}{c}\text { Relative } \\
\text { intensity (\%) }\end{array}$ & SD & cV & $\begin{array}{c}{[\mathrm{M}+\mathrm{Na}]^{+}} \\
m / z\end{array}$ & $\begin{array}{c}\text { Relative } \\
\text { intensity (\%) }\end{array}$ & SD & cV \\
\hline 1 & H3N4F1 & 5.79 & 19.90 & 0.02 & 0.12 & 1835.9 & 18.81 & 1.32 & 7.02 \\
\hline 2 & H3N5F1 & 6.14 & 4.39 & 0.03 & 0.77 & 2081.0 & 4.03 & 0.48 & 11.92 \\
\hline 3 & H4N4F1 & 6.52 & 20.47 & 0.07 & 0.34 & 2040.0 & & & \\
\hline 4 & H4N4F1 & 6.64 & 8.64 & 0.07 & 0.81 & 2040.0 & 30.41 & 1.83 & 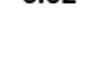 \\
\hline 5 & H4N5F1 & 6.79 & 4.36 & 0.03 & 0.74 & 2285.1 & 6.13 & 0.75 & 12.23 \\
\hline 6 & H5N4F1 & 7.37 & 14.63 & 0.03 & 0.18 & 2244.1 & 14.57 & 0.76 & 5.22 \\
\hline 7 & H5N5F1 & 7.66 & 2.47 & 0.02 & 0.86 & 2489.2 & 2.22 & 0.54 & 24.35 \\
\hline 8 & H5N4S1 & 7.99 & 2.12 & 0.06 & 2.92 & 2431.2 & 3.98 & 0.21 & 5.28 \\
\hline 9 & H5N4F1S1 & 8.34 & 10.91 & 0.03 & 0.32 & 2605.3 & 8.07 & 0.33 & 4.09 \\
\hline 10 & H5N5F1S1 & 8.58 & 2.65 & 0.02 & 0.63 & 2850.4 & 3.55 & 0.55 & 15.48 \\
\hline 11 & H5N4S2 & 8.98 & 1.39 & 0.08 & 5.66 & 2792.3 & 2.42 & 0.68 & 28.14 \\
\hline 12 & H5N4F1S2 & 9.3 & 3.74 & 0.02 & 0.66 & 2966.4 & 3.03 & 0.81 & 26.74 \\
\hline 13 & H5N5F1S2 & 9.44 & 4.05 & 0.10 & 2.55 & 3211.5 & 2.78 & 0.91 & 32.68 \\
\hline
\end{tabular}


Table S-2: Glycan composition, Rls, SDs and CVs for 6 human IgG N-glycan samples that were permethylated and analyzed on the same day using MALDI-TOF-MS to assess intraday variability.

\begin{tabular}{|c|c|c|c|c|}
\hline \multicolumn{5}{|c}{ MALDI-TOF-MS of permethylated human IgG } \\
\hline $\begin{array}{c}\text { Glycan } \\
\text { composition }\end{array}$ & $\begin{array}{c}\text { Relative } \\
\text { [M/Na] }]^{+}\end{array}$ & $\begin{array}{c}\text { intensity } \\
\text { (\%) }\end{array}$ & SD & CV \\
\hline H3N4F1 & 1835.9 & 17.83 & 0.98 & 5.49 \\
\hline H4N4F1 & 2040.0 & 28.64 & 1.53 & 5.33 \\
\hline H3N5F1 & 2081.1 & 4.22 & 0.91 & 21.62 \\
\hline H5N4F1 & 2244.1 & 13.39 & 0.67 & 5.04 \\
\hline H4N5F1 & 2285.1 & 5.83 & 0.65 & 11.16 \\
\hline H5N4S1 & 2431.2 & 3.49 & 0.53 & 15.28 \\
\hline H4N4F1S1 & 2401.2 & 3.09 & 0.43 & 14.00 \\
\hline H5N5F1 & 2489.2 & 1.74 & 0.35 & 20.35 \\
\hline H5N4F1S1 & 2605.3 & 9.08 & 0.71 & 7.85 \\
\hline H5N4S2 & 2792.3 & 3.23 & 0.45 & 13.85 \\
\hline H5N5F1S1 & 2850.4 & 2.65 & 0.58 & 21.80 \\
\hline H5N4F1S2 & 2966.4 & 3.48 & 0.51 & 14.78 \\
\hline H5N5F1S2 & 3211.5 & 3.32 & 0.34 & 10.17 \\
\hline
\end{tabular}


A

\begin{tabular}{|c|c|c|c|c|}
\hline \multicolumn{5}{|c|}{ MALI-TOF-MS of permethylated human IgG } \\
\hline $\begin{array}{c}\text { Glycan } \\
\text { composition }\end{array}$ & $\begin{array}{c}\text { [M+Na] } \\
\mathrm{m} / \mathrm{z}\end{array}$ & $\begin{array}{c}\text { Relative } \\
\text { intensity } \\
(\%)\end{array}$ & SD & $\mathrm{CV}$ \\
\hline H3N4F1 & 1835.9 & 18.02 & 2.55 & 14.16 \\
\hline H4N4F1 & 2040.0 & 28.63 & 4.03 & 14.08 \\
\hline H3N5F1 & 2081.1 & 4.48 & 2.11 & 47.03 \\
\hline H5N4F1 & 2244.1 & 13.68 & 1.56 & 11.40 \\
\hline H4N5F1 & 2285.1 & 6.19 & 2.57 & 41.53 \\
\hline H5N4S1 & 2431.2 & 3.33 & 1.57 & 47.14 \\
\hline H4N4F1S1 & 2401.2 & 3.14 & 1.75 & 55.55 \\
\hline H5N5F1 & 2489.2 & 1.91 & 1.25 & 65.45 \\
\hline H5N4F1S1 & 2605.3 & 8.12 & 2.06 & 25.42 \\
\hline H5N4S2 & 2792.3 & 3.74 & 2.45 & 65.40 \\
\hline H5N5F1S1 & 2850.4 & 2.36 & 1.14 & 48.13 \\
\hline H5N4F1S2 & 2966.4 & 3.10 & 1.68 & 54.09 \\
\hline H5N5F1S2 & 3211.5 & 3.30 & 1.54 & 46.47 \\
\hline
\end{tabular}

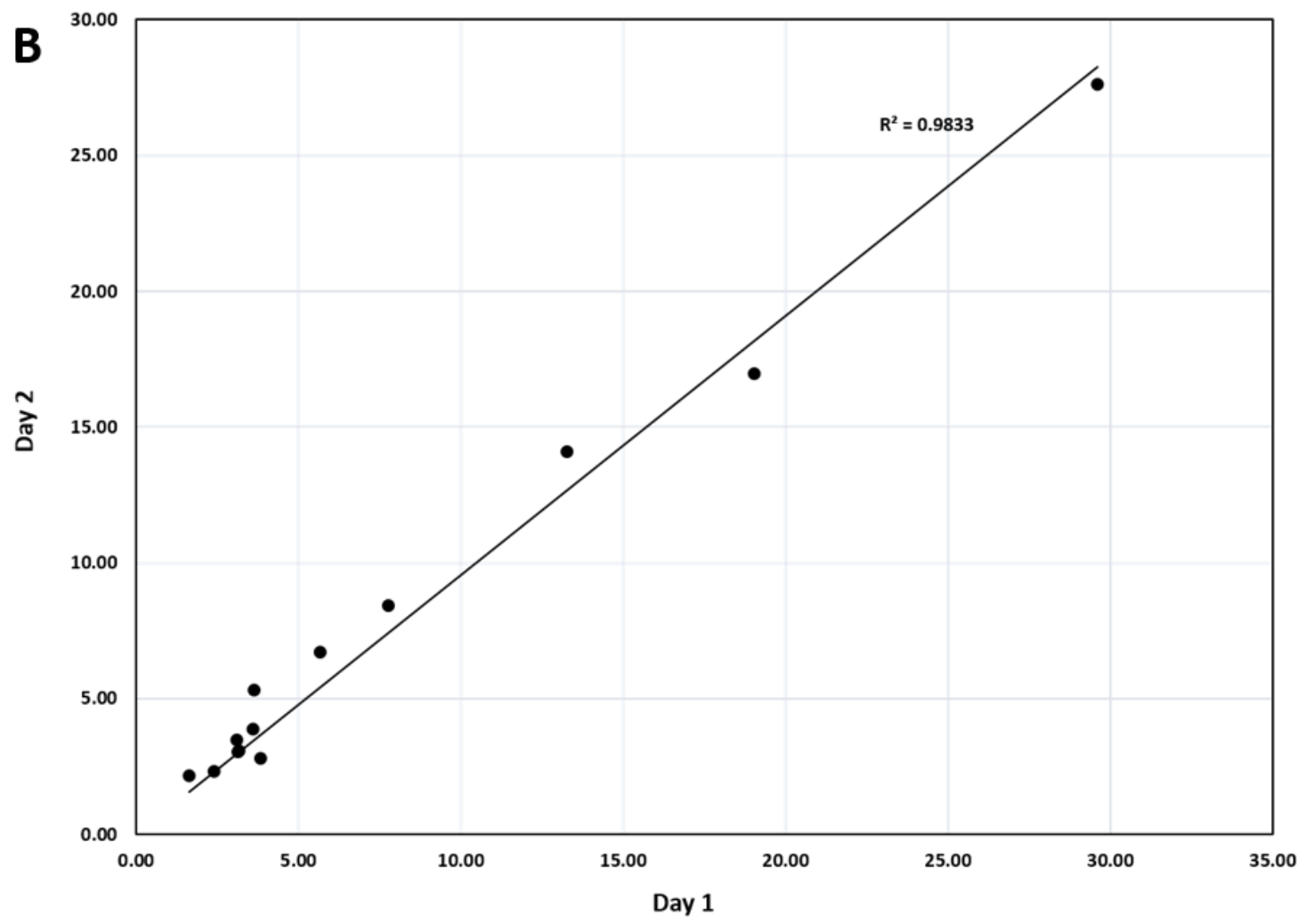

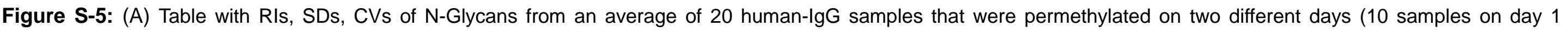

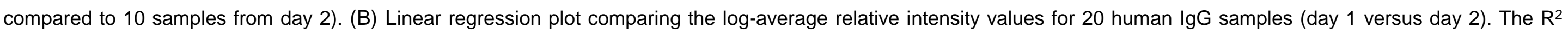
value $=0.98$, which is a value close to 1 and therefore shows a good correlation and a low variation between the two data sets. 


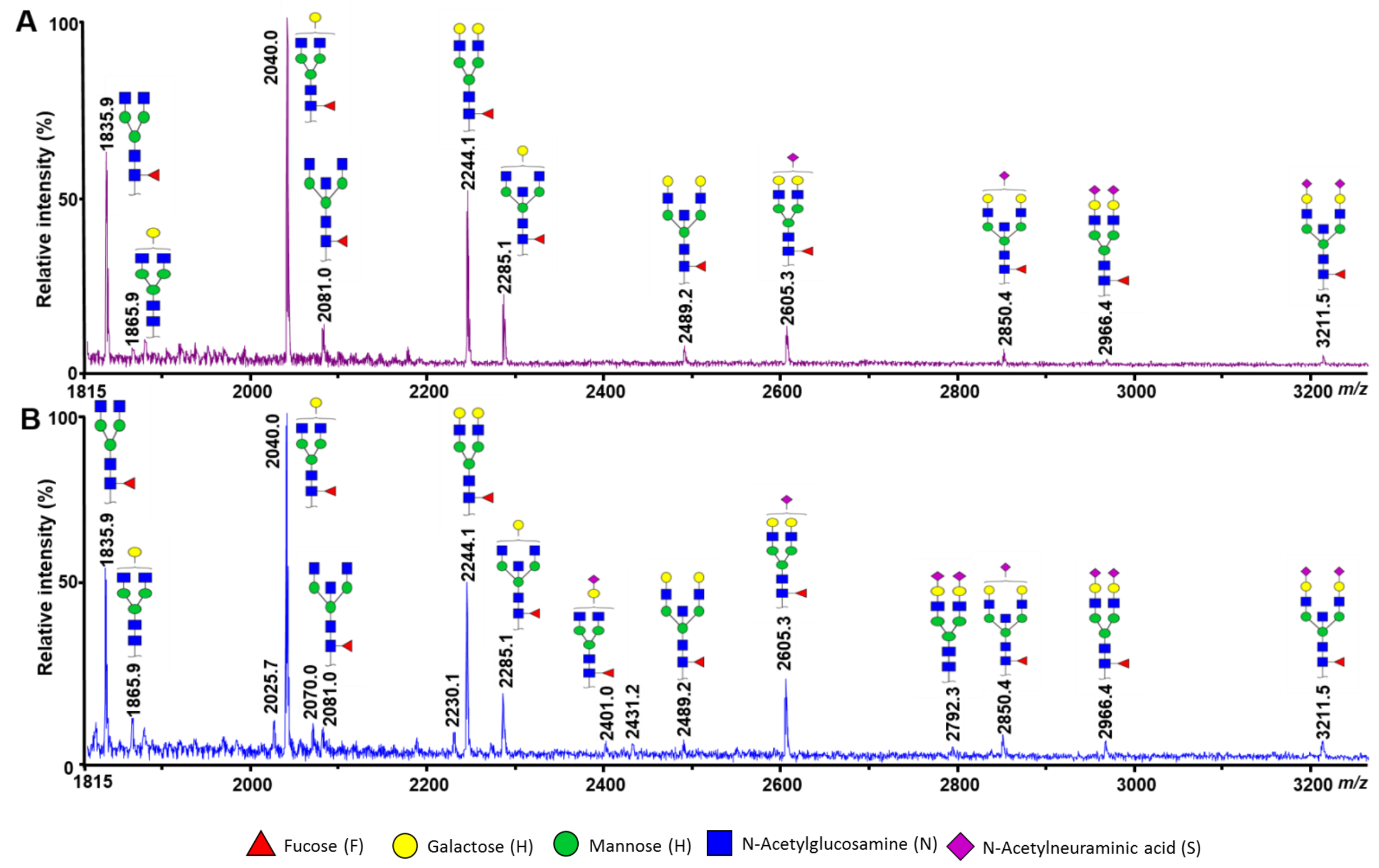

Figure S-6: MALDI-TOF-MS spectra of permethylated N-glycans from human IgG. (A) In-solution permethylation was performed manually which included longer sample preparation times along with labor intensive and repetitive extraction steps. (B) Automated high throughput permethylation was performed on the liquid handling robot eliminating manual extraction steps and faster processing times. The automated method provides comparable results to the manual in-solution method. Structures for glycans are depicted following the CFG notation: $N$-acetylglucosamine (blue square), fucose (red triangle), mannose (green circle), galactose (yellow circle), and $\mathrm{N}$-acetylneuraminic acid (purple diamond). 

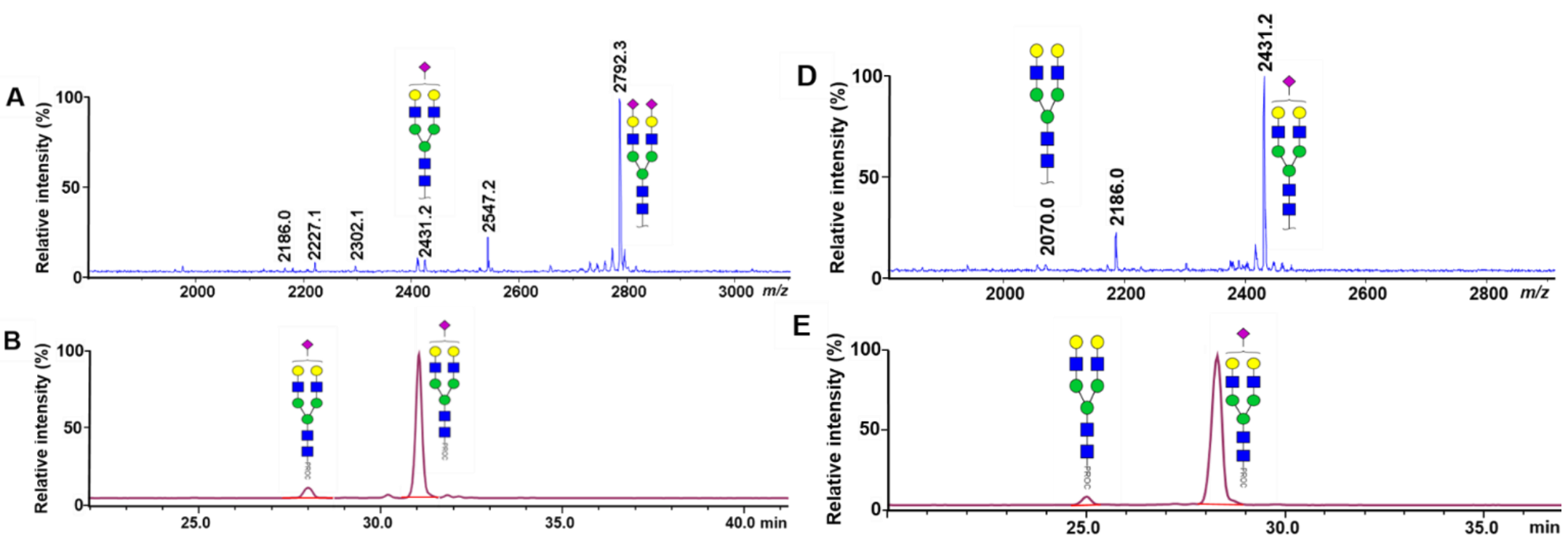

C

\section{$100-$ HILIC UHPLC}

80

60

40

0

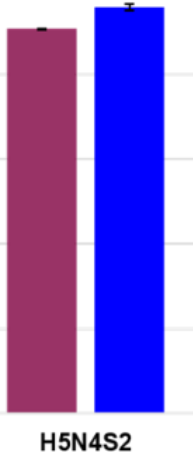

F

100

- HILIC UHPLC

80

60

0

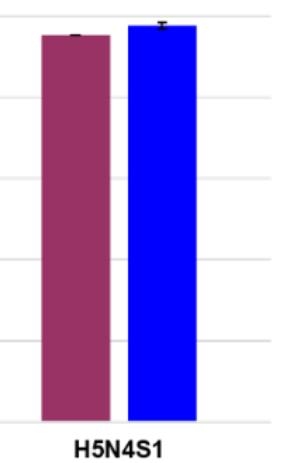

H5N4S1

H5N4S2

H5N4

H5N4S1

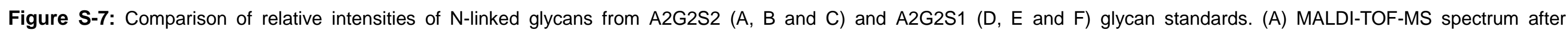

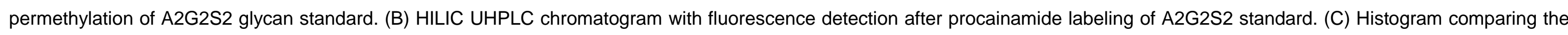

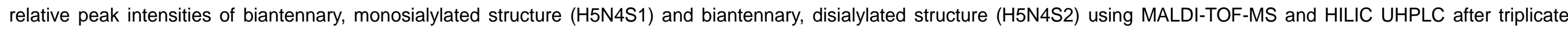

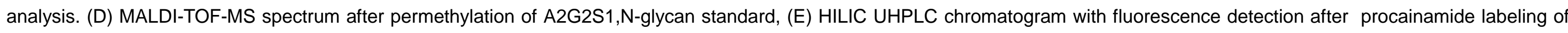

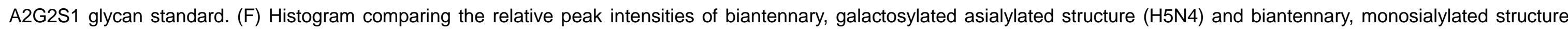
(H5N4S1) using MALDI-TOF-MS and HILIC UHPLC after triplicate analysis. Samples were permethylated and procainamide labeled using the liquid handling robot. 


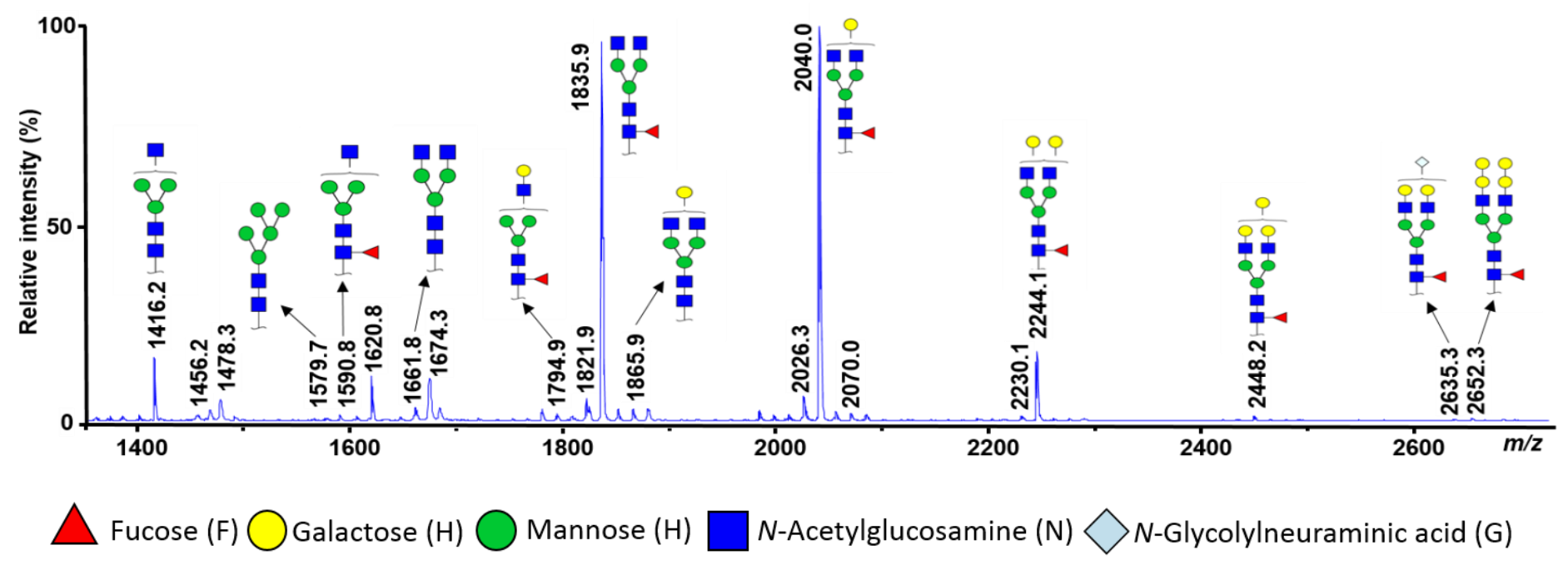

Figure S-8A: MALDI-TOF-MS spectrum of IgG1 mAb N-glycans permethylated on the liquid handling robot. The $\mathrm{N}$-glycan structures in the spectrum were established and peak assignments were confirmed through data obtained from procainamide labeling and exoglycosidase digestion of the $\mathrm{mAb}$. 


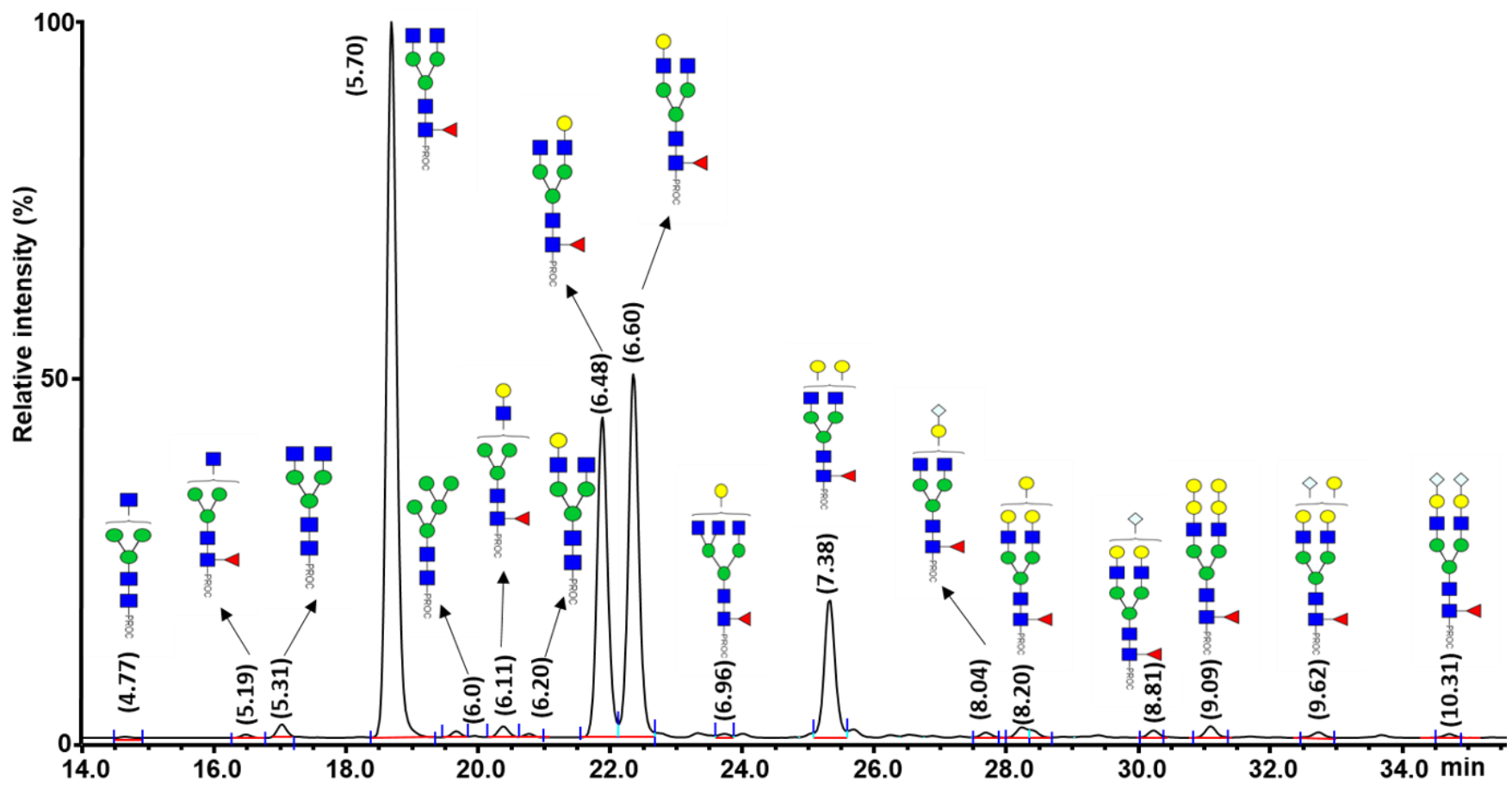

Figure S-8B: UHPLC profile of procainamide labeled IgG1 N-glycans. (Note: Measuring 96 samples on UHPLC takes a minimum of 48 hours). 
Table S-3: Bioreactor culture condition, glycan compositions, RIs, SDs, CVs for 2-AB labeled (HILIC UHPLC) data and permethylated (MALDI-TOF-MS) data of N-glycans from IgG4 mAbs.

\begin{tabular}{|c|c|c|c|c|c|c|c|}
\hline \multirow{2}{*}{$\begin{array}{l}\text { Culture } \\
\text { condition }\end{array}$} & \multirow{2}{*}{$\begin{array}{c}\text { Glycan } \\
\text { composition }\end{array}$} & \multicolumn{3}{|c|}{$\begin{array}{l}\text { 2-AB labeled } \\
\text { HILIC UHPLC }\end{array}$} & \multicolumn{3}{|c|}{$\begin{array}{l}\text { Permethylated } \\
\text { MALDI-TOF-M S }\end{array}$} \\
\hline & & $\begin{array}{c}\text { Relative } \\
\text { intensity } \\
(\%) \\
\end{array}$ & SD & cv & $\begin{array}{c}\text { Relative } \\
\text { intensity } \\
(\%)\end{array}$ & SD & $\mathrm{cv}$ \\
\hline \multirow{3}{*}{ DGS } & H3N4F1 & 51.6 & 0.67 & 1.31 & 49.8 & 2.39 & 4.80 \\
\hline & H4N4F1 & 38.0 & 0.84 & 2.22 & 41.5 & 1.45 & 3.50 \\
\hline & H5N4F1 & 10.3 & 0.17 & 1.71 & 8.6 & 0.94 & 10.82 \\
\hline \multirow{3}{*}{ SMA } & H3N4F1 & 52.7 & 0.22 & 0.42 & 51.9 & 1.05 & 2.02 \\
\hline & H4N4F1 & 36.3 & 0.30 & 0.83 & 38.6 & 1.11 & 2.88 \\
\hline & H5N4F1 & 10.7 & 0.09 & 0.88 & 9.5 & 0.37 & 4.00 \\
\hline \multirow{3}{*}{ scc } & H3N4F1 & 55.0 & 0.17 & 0.30 & 54.3 & 1.16 & 2.14 \\
\hline & H4N4F1 & 37.7 & 0.14 & 0.40 & 39.2 & 0.94 & 2.40 \\
\hline & H5N4F1 & 7.3 & 0.06 & 0.88 & 6.4 & 0.41 & 6.43 \\
\hline \multirow{3}{*}{ HCC } & H3N4F1 & 60.0 & 0.12 & 0.21 & 61.0 & 2.77 & 4.54 \\
\hline & H4N4F1 & 33.7 & 0.15 & 0.44 & 33.0 & 1.76 & 5.36 \\
\hline & H5N4F1 & 6.3 & 0.03 & 0.56 & 6.0 & 1.08 & 18.25 \\
\hline \multirow{3}{*}{ CTC } & H3N4F1 & 63.3 & 0.13 & 0.20 & 65.1 & 0.76 & 1.17 \\
\hline & H4N4F1 & 31.3 & 0.08 & 0.25 & 30.5 & 0.55 & 1.80 \\
\hline & H5N4F1 & 5.2 & 0.04 & 0.90 & 4.4 & 0.24 & 5.50 \\
\hline
\end{tabular}

Key to bioreactor conditions: DGS - Direct Gas Sparging; SMA - Silicone Membrane Aeration; SCC - 

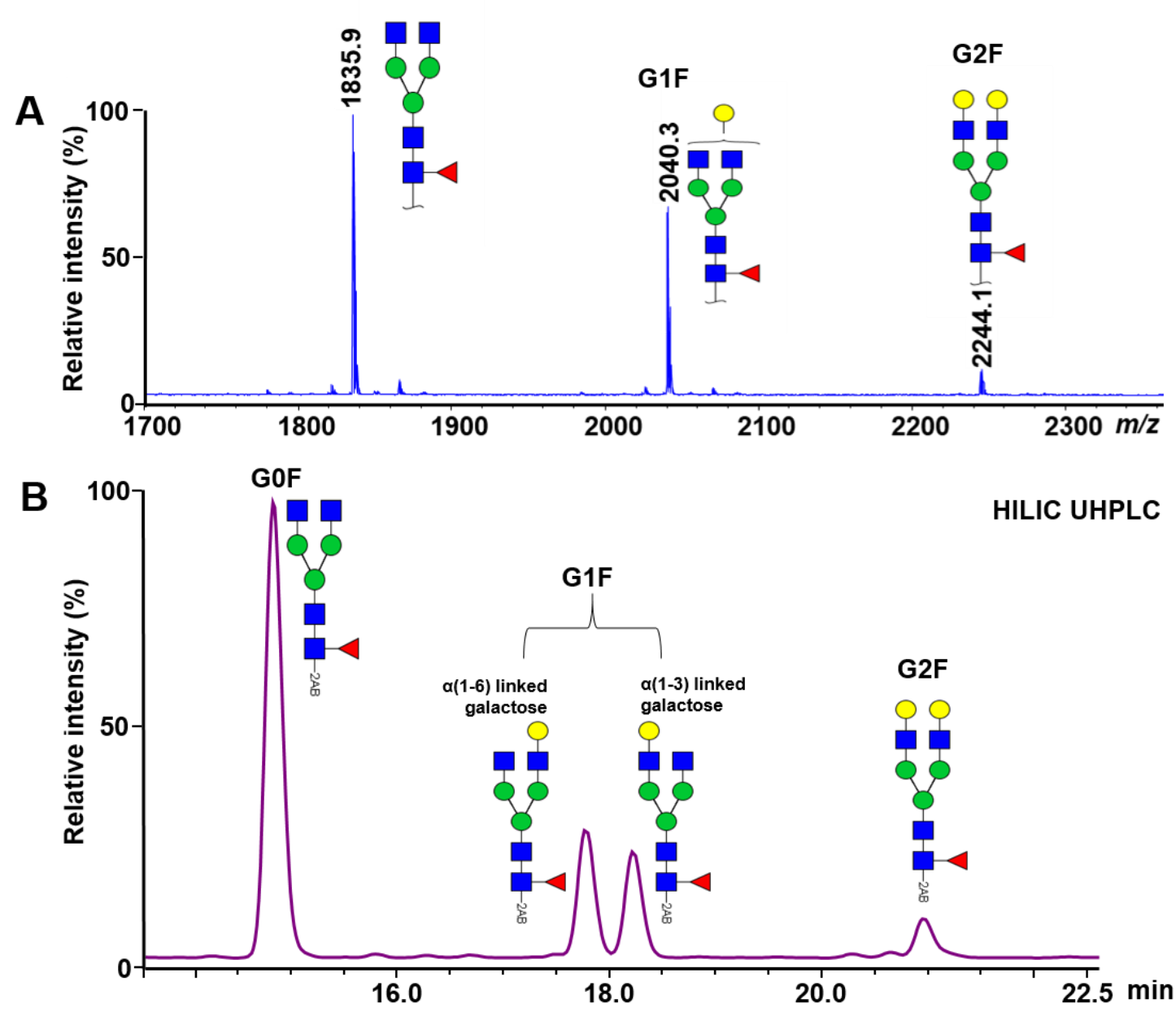

Figure S-9: Typical N-glycan trace from IgG4 mAb grown in stirred tank bioreactor with varied culture conditions (A) MALDI-TOF-MS analysis of IgG4 mAb after automated permethylation (B) HILIC UHPLC chromatogram of IgG4 mAb after automated 2-AB labeling. The isobaric G1F structures are not distinguished in the simple MALDITOF-MS profile while the HILIC UHPLC chromatogram clearly depicts different structural distribution of galactose. 

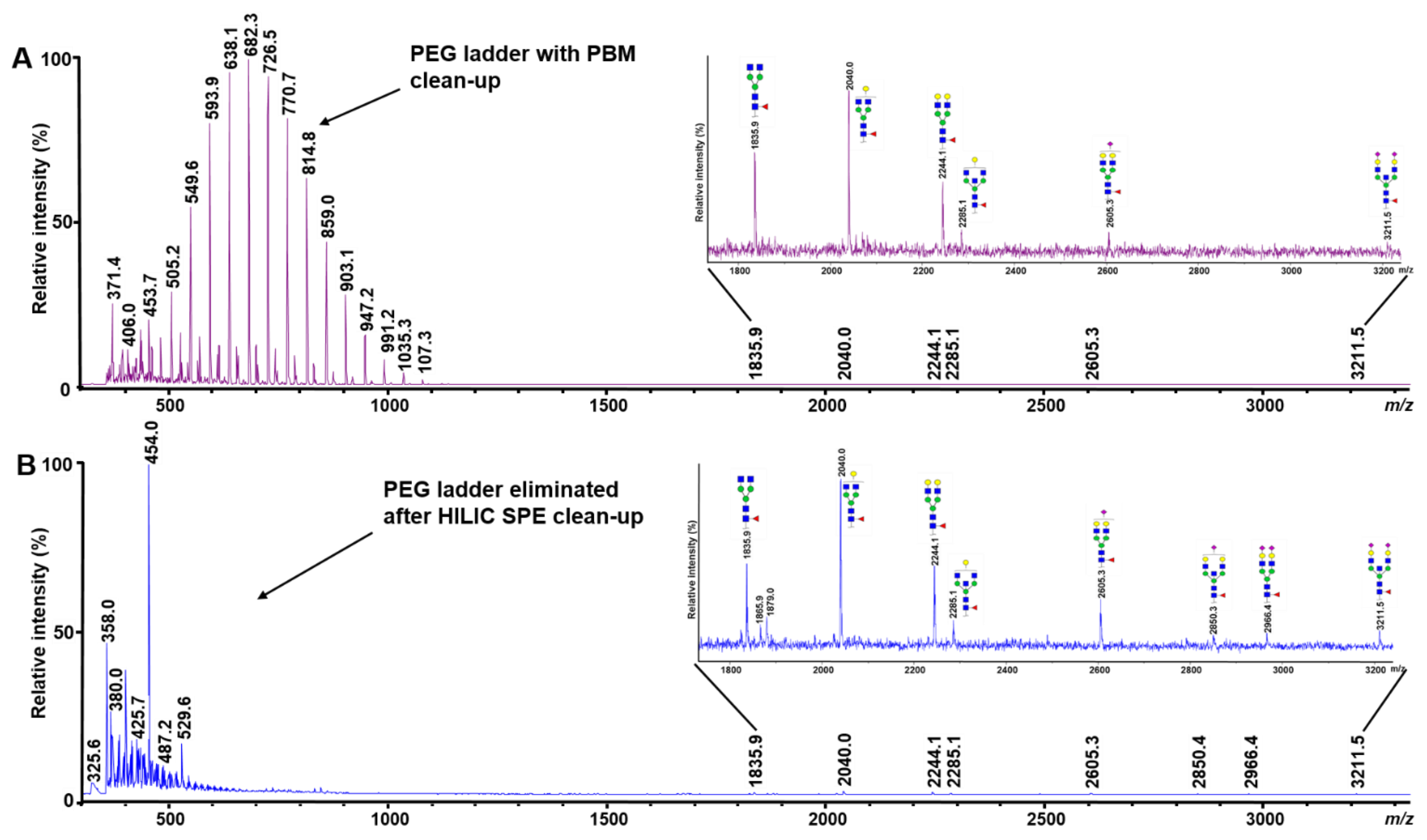

Figure S-10: Comparison of PNGase F released human IgG N-glycans with protein binding membrane (PBM) clean-up and with Hydrophilic Liquid Interaction Chromatography- Solid Phase extraction (HILIC SPE) clean-up with $Y$ axis normalized. (A) Permethylated MALDI-TOF-MS of human IgG N-glycans after automated enzymatic release and PBM clean-up, showing a large polyethylene glycol (PEG) ladder. (B) Permethylated MALDI-TOF-MS of human IgG N-glycans after automated enzymatic release with HILIC SPE clean-up showing elimination of PEG ladder and salts. 


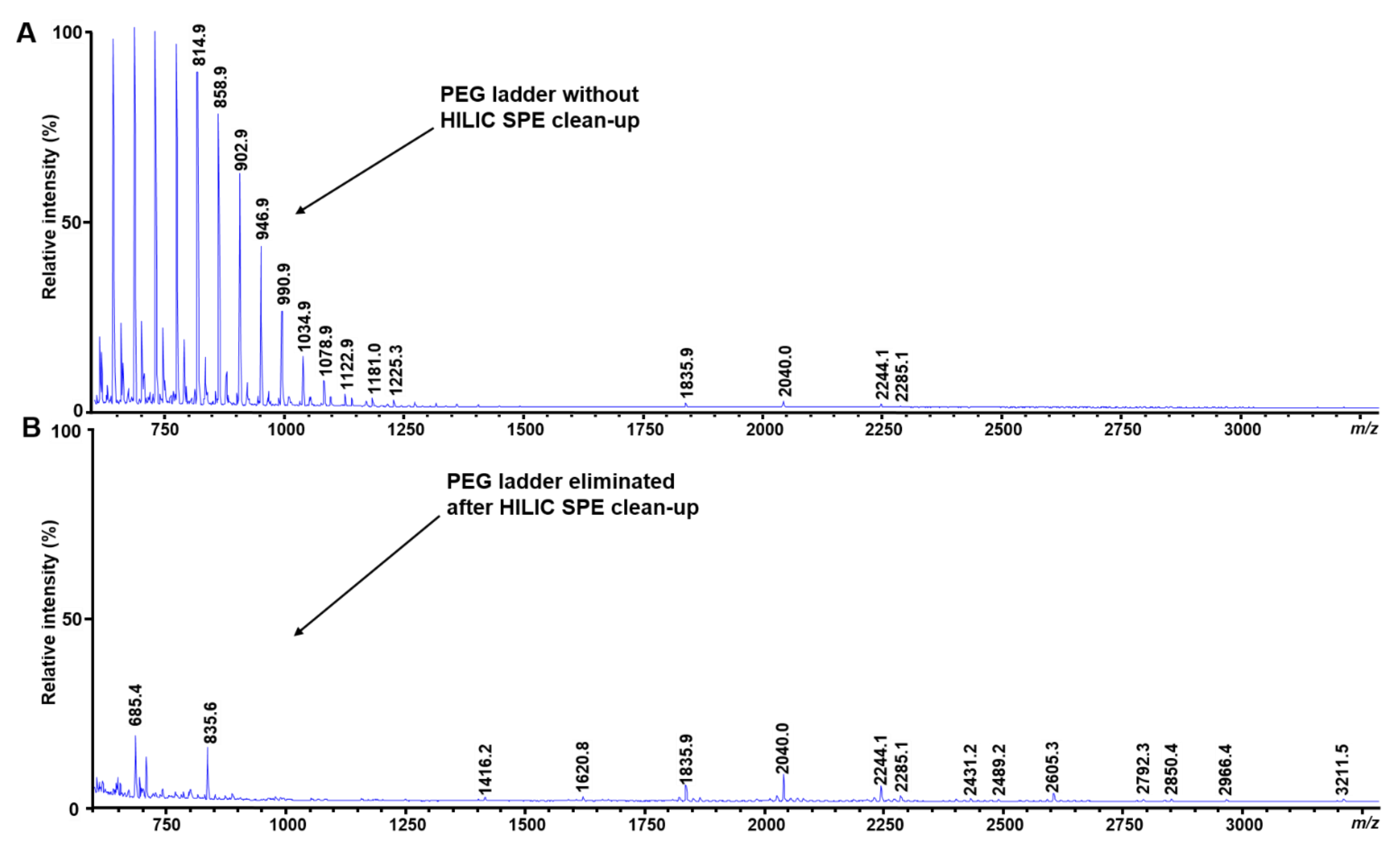

Figure S-11: Comparison of PNGase F released human IgG N-glycans with and without Hydrophilic Liquid Interaction Chromatography-Solid Phase extraction (HILIC SPE) clean-up where Y axis is normalized. (A) Permethylated MALDI-TOF-MS of human IgG N-glycans after automated enzymatic release and no HILIC SPE clean-up, showing a large polyethylene glycol (PEG) ladder. (B) Permethylated MALDI-TOF-MS of human IgG N-glycans after automated enzymatic release with HILIC SPE clean-up, showing elimination of PEG ladder and salts. 


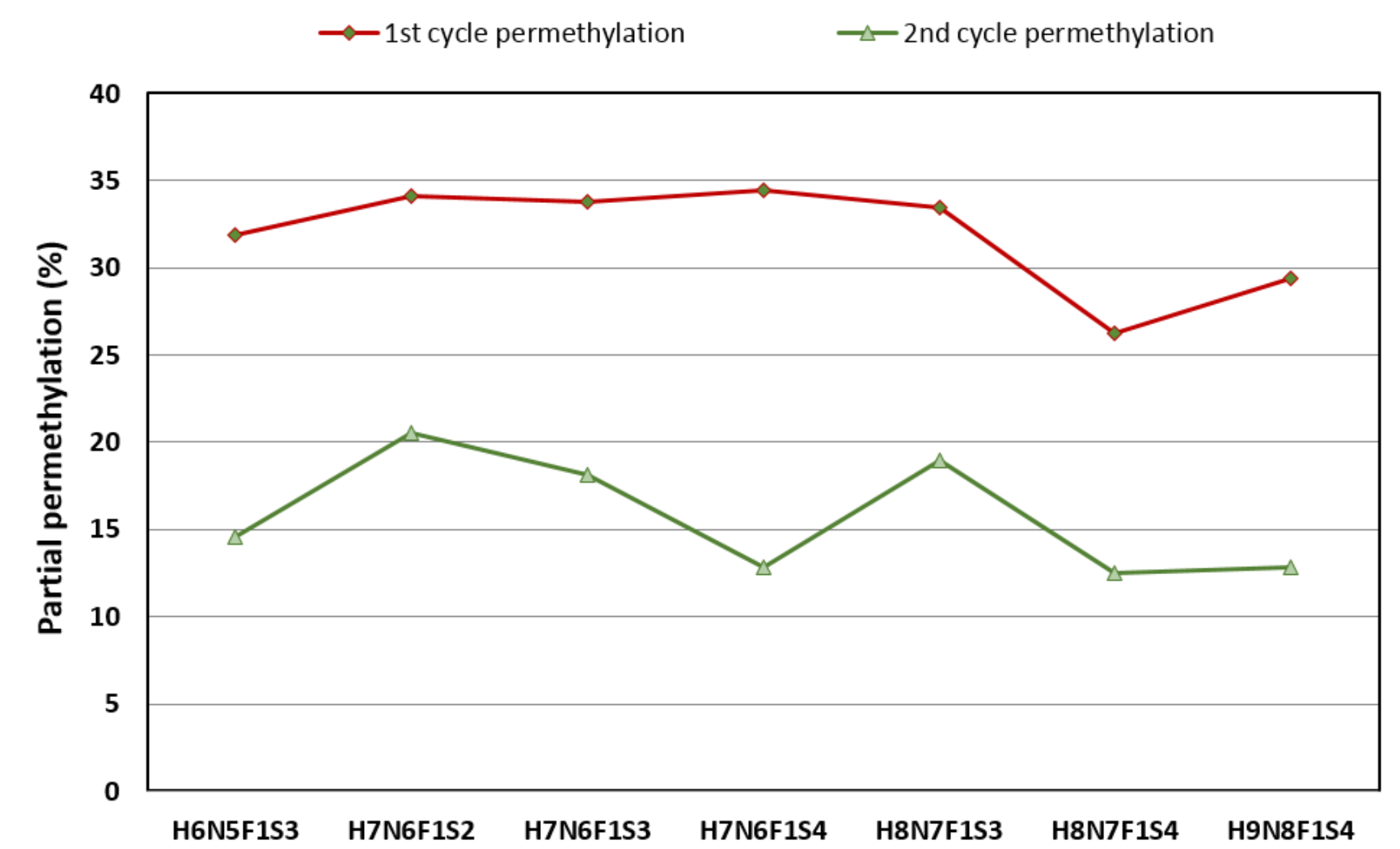

$\mathrm{N}$-glycan compositions

Figure S-12: Efficiency of automated HT permethylation as a function of reduction of partial permethylation after two cycles of derivatization for $\mathrm{N}$-glycans derived from rhEPO. 
30

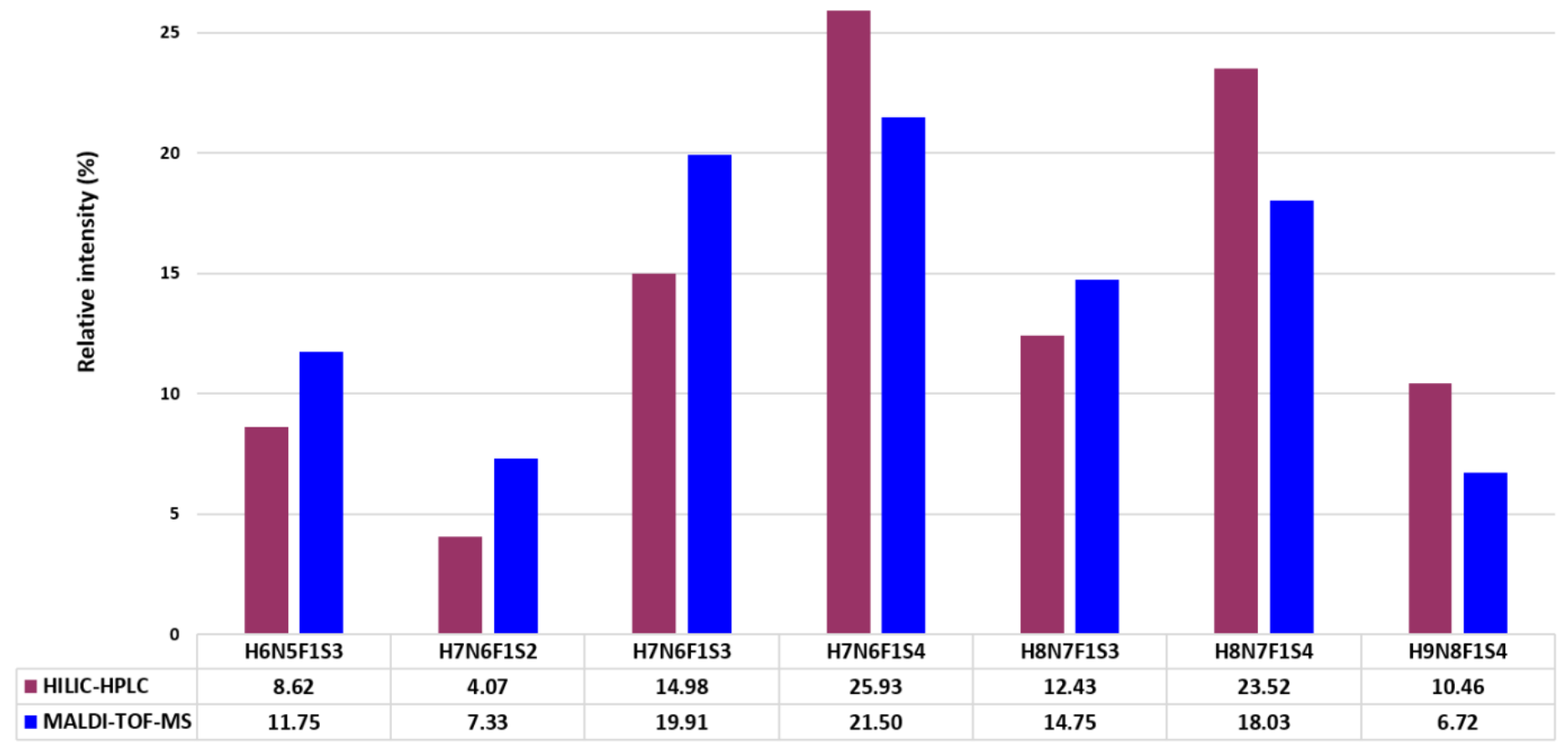

Figure S-13: Histogram comparing the relative intensities of major $7 \mathrm{~N}$-linked glycans from rhEPO with mauve bars representing HILIC-HPLC data and blue bars depicting MALDI-TOF-MS data. 